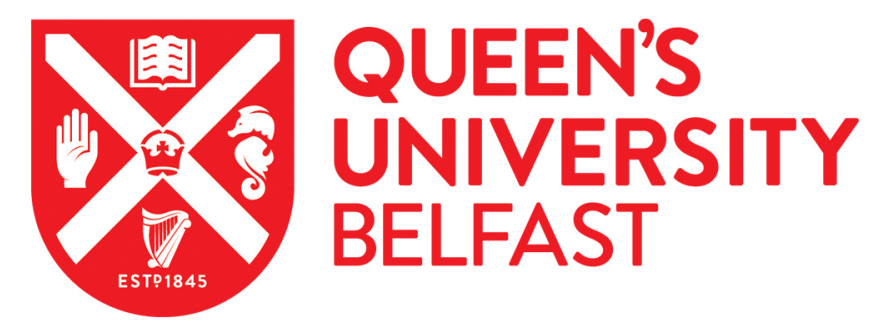

\title{
Strain Engineering and Raman Spectroscopy of Monolayer Transition Metal Dichalcogenides
}

Dadgar, A. M., Scullion, D., Kang, K., Esposito, D., Yang, E. H., Herman, I. P., Pimenta, M. A., Santos, E. J. G., \& Pasupathy, A. N. (2018). Strain Engineering and Raman Spectroscopy of Monolayer Transition Metal Dichalcogenides. Chemistry of Materials, 1-8. https://doi.org/10.1021/acs.chemmater.8b01672

Published in:

Chemistry of Materials

Document Version:

Peer reviewed version

Queen's University Belfast - Research Portal:

Link to publication record in Queen's University Belfast Research Portal

Publisher rights

(c) 2018 American Chemical Society. This work is made available online in accordance with the publisher's policies. Please refer to any applicable terms of use of the publisher.

\section{General rights}

Copyright for the publications made accessible via the Queen's University Belfast Research Portal is retained by the author(s) and / or other copyright owners and it is a condition of accessing these publications that users recognise and abide by the legal requirements associated with these rights.

Take down policy

The Research Portal is Queen's institutional repository that provides access to Queen's research output. Every effort has been made to ensure that content in the Research Portal does not infringe any person's rights, or applicable UK laws. If you discover content in the Research Portal that you believe breaches copyright or violates any law, please contact openaccess@qub.ac.uk. 


\section{Strain Engineering and Raman Spectroscopy of}

\section{Monolayer Transition Metal Dichalcogenides}

Abdollah M. Dadgar ${ }^{1,2}$, Declan Scullion ${ }^{3}$, Kyungnam Kang ${ }^{4}$, Daniel Esposito ${ }^{5}$, Eui-Hyoek Yang $^{4}$, Irving P. Herman ${ }^{6}$, Marcos A. Pimenta ${ }^{7}$, Elton J. G. Santos ${ }^{8}$, Abhay N. Pasupathy ${ }^{2}$

${ }^{1}$ Department of Mechanical Engineering, Columbia University, New York, NY 10027, USA

${ }^{2}$ Physics Department, Columbia University, New York, NY, 10027, USA

${ }^{3}$ School of Mathematics and Physics, Queens University, Belfast, BT7 1NN, UK

${ }^{4}$ Department of Mechanical Engineering, Stevens Institute of Technology, Castle Point on the Hudson, Hoboken, NJ, USA

${ }^{5}$ Department of Chemical Engineering, Columbia University - New York, NY 10027, USA

${ }^{6}$ Department of Applied Physics and Applied Mathematics, Columbia University, New York NY 10027, USA

${ }^{7}$ Department of Physics, Universidade Federal de Minas Gerais (UFMG), Brazil

${ }^{8}$ School of Chemistry and Chemical Engineering, Queen's University - Belfast, BT9 5AL, UK 
ABSTRACT: We describe a facile technique based on polymer encapsulation to apply several percent $(>5 \%)$ controllable strains to monolayer and few-layer Transition Metal Dichalcogenides (TMDs). We use this technique to study the lattice response to strain via polarized Raman spectroscopy in monolayer $\mathrm{WSe}_{2}$ and $\mathrm{WS}_{2}$. The application of strain causes mode-dependent redshifts, with larger shift rates observed for in-plane modes. We observe a splitting of the degeneracy of the in-plane $E^{\prime}$ modes in both materials and measure the Grüneisen parameters. At large strain, we observe that the reduction of crystal symmetry can lead to a change in the polarization response of the $A^{\prime}$ mode in $\mathrm{WS}_{2}$. While both $\mathrm{WSe}_{2}$ and $\mathrm{WS}_{2}$ exhibit similar qualitative changes in the phonon structure with strain, we observe much larger changes in mode positions and intensities with strain in $\mathrm{WS}_{2}$. These differences can be explained simply by the degree of iconicity of the metal-chalcogen bond.

One of the iconic characteristics of monolayer 2D materials is their elasticity, which allows them to be subjected to several percent strain before yielding [1]. The application of moderate $(\sim 1 \%)$ strains is expected to change the anharmonicity of interatomic potentials $[2,3]$, phonon frequencies $[4,5]$ and effective masses [6, 7]. At larger strains, topological electronic [8, 9] and semiconductor-metal structural phase changes have been predicted [10-13]. Important technological applications such as piezoelectricity can be explored by the application of systematic strain [14-16]. One of the chief problems in achieving reproducible strain is the intrinsic nature of 2D materials as single layer sheets - they need to be held to a flexible substrate which is then stretched or compressed. Previous experiments [17-20] have used flexible polymers as substrates and metal or polymer caps in order to constrain the $2 \mathrm{D}$ material. Using 
these techniques, approximate strains up to $4 \%$ have been reported so far in the literature, but independent verification of the applied strain has been lacking. In detailed studies of strain transfer between polymers and 2D materials, slipping between the two has been identified as a key issue [21]. Achieving large reproducible strains in engineered geometries will allow us to probe these exciting properties of individual 2D materials and their heterostructures $[4,18,22-$ $28]$.

In this work, we develop a new strain platform to apply large range accurate uniaxial tensile strains on monolayer and few-layer materials. One of our chief innovations is the development of a novel polymer-based encapsulation method to enable the application of large strain to 2D materials. Here, we apply this technique to study the strain-dependent properties of monolayer $\mathrm{WSe}_{2}$ and $\mathrm{WS}_{2}$ grown by Chemical Vapor Deposition (CVD) on $\mathrm{SiO}_{2} / \mathrm{Si}$ substrates [29-31]. We use cellulose acetate butyrate $(\mathrm{CAB})$ to lift the monolayers from the $\mathrm{SiO}_{2} / \mathrm{Si}$ substrates and transfer to polycarbonate (PC) substrates. The two polymers are then bonded to produce encapsulated monolayers and multilayers. The key to achieving good bonding is perfect control over the temperature, time and pressure during the bonding process. Additionally, polymer layers that are in the amorphous phase cause nonlinear strain-deflection behavior which is not desirable in our experiments. To resolve this issue, we crystallize the polymer stacks by annealing near the glass transition temperature followed by slow cooling. First, we measured the glass transition temperature $\left(\mathrm{T}_{\mathrm{g}}\right)$ of each polymer blend we use, by continuously heating it on an accurate PIDcontrolled hotplate $\left( \pm 0.1^{\circ} \mathrm{C}\right.$ accuracy) while checking its ductility. After determining $\mathrm{T}_{\mathrm{g}}$, we assemble the polymer stack and perform the bonding process at $\mathrm{T}_{\mathrm{B}}=0.97 \mathrm{~T}_{\mathrm{g}}$. We use a compressive pressure of 3.0 psi for 60 min to complete the bonding, followed by slow cooling at a rate of $2 \mathrm{~K} / \mathrm{min}$ to room temperature. We find that this process of encapsulation does not 
modify the peaks or intensity ratios in the Raman spectra, indicating that the structural qualities of the films are maintained during the encapsulation process. To additionally check the electronic properties of the bonded film, we perform photoluminescence (PL) spectroscopy of the monolayers after growth and after encapsulation. We find from the PL measurements that the asgrown films on $\mathrm{SiO}_{2}$ are under strain. Upon release from the $\mathrm{SiO}_{2}$ substrate, this strain is released. No additional strain is produced during the bonding process, and we also do not see any evidence for chemical modification of the films during bonding. Further information on the PL spectra is available in the Supporting Information document. The crystallized polymers are fully flexible, elastic and springy substrates as shown in Fig. 1(a). After all of our processing steps, we find that the polymer stacks enter into the plastic regime at $7 \%$ strain. We find that strains up to this value are perfectly transferred to the encapsulated 2D material as described below.

Our strain method adopts the extra-neutral axis bending technique - Fig. 1(b) in which areas above the neutral axis undergo tensile strain while those below the axis experience compressive strain. In our method, we use a screw-driven vertical translation stage to apply strain to the polymer stacks. We solve the Euler-Bernoulli equation for our geometry in order to achieve an accurate relation between the vertical displacement $\delta$ of the translation stage and the strain $\varepsilon$ of the 2D material. For a fully isotropic, linear and elastic material, the strain-displacement relation is derived as: $\varepsilon=3 t \delta / a(3 b+2 a)$ where $t$ is the substrate thickness, $b$ and $a$ are center support and cantilever lengths respectively. In our experiments, the use of a fine adjustment screw gives us a resolution of $0.05 \%$ strain for $0.5 \mathrm{~mm}$ substrates, with essentially no limit to the maximum strain that can be applied. More details are provided in the Supplemental Material.

Shown in Fig. 1(c) is an optical image of triangular flakes of WSe2 encapsulated by this process. We adjust the CVD process to produce triangular flakes in order to easily identify the 
crystallographic directions of the grown monolayers. Since the strains achievable in our experiments are large, we can directly verify from optical measurements that the strain being applied to 2D layer is the calculated value. This is illustrated in Fig. 1(d). Each of these images is obtained by overlaying two images, one at zero strain and one at a fixed value of strain $(4.2 \%$ and $6.5 \%$ respectively). Only the edges of the triangles are shown in the images, which are lined up to be at the same vertical height at the top vertex of the triangle. We can directly see by inspection that the length of the triangle along the strain direction is larger when strained as one expects. A pixel-height measurement of the edge-detected images gives us a direct experimental measure of the applied strain, which can be compared to the calculated strain based on the screw displacement. It is found that the two measurements match within $0.1 \%$ absolute strain. We have also confirmed by direct measurement that the strain produced over the entire length $b$ is uniform within our measurement accuracy as long as we remain in the elastic limit of our polymers. Thus, our technique allows for the application of uniform, highly repeatable and independently measurable strain on TMD monolayers and heterostructures.

\section{Results and Discussions}

In order to probe the effects of strain on our samples, we choose to characterize with Raman spectroscopy - a simple yet powerful way to measure lattice properties and their coupling to the electronic degrees of freedom. Strains were applied in both zigzag and armchair directions (Y and $\mathrm{X}$ axes in Fig. 1(e) ) in our experiments. Our Raman setup with $532 \mathrm{~nm}$ excitation wavelength is shown in Fig. 1 (f). The measurements were performed while controlling for the incident light's polarization $\left(E_{i}\right)$ direction $(\theta$ in Fig. 1(e) ). For each experiment, Raman spectra were collected in both the parallel- $\left(E_{s} \| E_{i}\right)$ and cross-polarized $\left(E_{s} \perp E_{i}\right)$ detector geometries, shown with standard notations $Z(Y Y) \bar{Z}$ and $Z(Y X) \bar{Z}$ respectively. In our experiments, we found 
no dependence of the Raman spectra on the angle of incidence relative to the crystallographic axis at zero strain. We therefore fix our incidence angle of $E_{i}$ to the $Y$ direction, and measure the unpolarized, parallel-polarized and cross-polarized Raman spectra at each value of strain which is applied in the $X$ direction.

We first discuss the properties of monolayer WSe2. Shown in Fig. 2(a) are a sequence of spectra taken at different values of strain in the unpolarized, parallel and cross polarization geometries. Previous Raman spectroscopy measurements performed on monolayer $\mathrm{WSe}_{2}$ have identified three vibrational modes [32-34] termed $A^{\prime}, E^{\prime}$ and $2 L A . A^{\prime}$ is an out-of-plane phonon mode in which the top and bottom chalcogen atoms vibrate in opposing directions; while $E^{\prime}$ is in-plane mode where the metal atoms vibrate out-of-phase with the chalcogen atoms [35]. The $2 L A$ mode results from a double resonance process involving two phonons from the $L A$ branch. Second order processes can in general give rise to a complex lineshape in the Raman spectrum; yet, in the case of $\mathrm{WSe}_{2}$ we find that a single Lorentzian can be used to model well the $2 L A$ mode lineshape. Although $A^{\prime}$ and $E^{\prime}$ modes are nearly degenerate, they can be distinguished from each other by polarization dependency of their intensities. The out of plane, symmetric $A^{\prime}$ mode disappears due to its symmetry in the cross polarization geometry, leaving behind only the $E^{\prime}$ mode. Our spectra in the cross-polarization geometry can thus be modeled well as the sum of two Lorentzian peaks corresponding to $E^{\prime}$ and $2 L A$ modes. Information of the $E^{\prime}$ mode position can then be used to fit the spectra seen in the parallel polarization geometry in order to extract the nearly-overlapping $A^{\prime}$ mode position.

Having understood the polarization-dependent Raman spectra of unstrained monolayer WSe2, we apply uniaxial strains and measure the Raman response. The effects of uniaxial strain up to $1 \%$ on monolayer $\mathrm{WSe}_{2}$ has previously been experimentally investigated via unpolarized Raman 
[18] and absorption spectroscopy [36]. Raman spectra under increasing uniaxial strain up to $3 \%$ are shown in Fig. 2(a). A close examination of spectral lineshapes in the cross polarization geometry shows that the $E^{\prime}$ mode becomes broader with increasing strain. In general, we expect that the initially doubly degenerate $E^{\prime}$ mode splits on the application of strain into $E^{\prime+}$ and $E^{\prime-}$. The displacement eigenvector of the $E^{\prime+}$ mode is orthogonal to the direction of strain, while it is parallel for the $E^{\prime-}$ mode, as has previously been observed for MoS2 and graphene $[3,17,23]$. While we cannot observe a complete separation of the $E^{\prime+}$ and $E^{\prime-}$ modes in our data, it is nevertheless straightforward to fit the lineshape to two Lorentzian functions and extract the splitting as a function of strain, as shown in Fig. 2(e). The splitting of the $E^{\prime}$ mode under tensile strain due to the anharmonictiy of molecular potentials can be described by Grüneisen parameter $\gamma$ and shear deformation potential $\beta$ :

$$
\begin{gathered}
\gamma=\frac{\left|\Delta \omega_{\mathrm{E}^{\prime+}}\right|+\left|\Delta \omega_{\mathrm{E}^{\prime-}}\right|}{2 \omega_{\mathrm{E}^{\prime}}(1-\mathrm{v})} \\
\beta=\frac{|| \Delta \omega_{\mathrm{E}^{\prime+}}|-| \Delta \omega_{\mathrm{E}^{\prime-}}||}{2 \omega_{\mathrm{E}^{\prime}}(1+\mathrm{v})}
\end{gathered}
$$

where $\omega_{E^{\prime}}$ is the frequency of $E^{\prime}$ mode, $\Delta \omega_{E^{\prime+}}$ and $\Delta \omega_{E^{\prime-}}$ are the frequency shifts of split modes per unit percent strain and $v$ is Poisson's ratio which is 0.27 for our substrates. We obtain values of $=0.38, \beta=0.10$ for $\mathrm{WSe}_{2}$ which are smaller than those reported for graphene $[2,3]$. Using the fits for $E^{\prime}$ mode from the cross-polarization geometry, we then extract the behavior of the $A^{\prime}$ mode as a function of strain. We find that this mode expectedly does not shift significantly with strain due to its nature as an out-of-plane excitation. Finally, the $2 L A$ mode can be easily fit in both parallel and cross polarization spectra. Small redshifts are observed in its position with increasing strain. We also observe an increase in the width of the $A^{\prime}$ and $2 L A$ modes with 
increasing strain, as well as a decrease in the intensities of all observed modes at higher strains. These observations are summarized in Fig. 2(d).

The spectra of WS 2 show additional structure [37], as can be seen in Fig. 3(a). Firstly, two additional lines that we term $P_{1}$ and $P_{2}$ are observed in the spectra at 303 and $332 \mathrm{~cm}-1$ respectively. We clarify the mode assignment of these peaks based on theory as described below. The $A^{\prime}$ mode is well separated from the other modes and is located at $423 \mathrm{~cm}-1$. Secondly, the region between $345 \mathrm{~cm}-1$ and $365 \mathrm{~cm}-1$ shows a complex lineshape. Previous measurements have shown that the $2 L A$ and $E^{\prime}$ modes are nearly degenerate in $\mathrm{WS}_{2}$. However, we find that even two Lorentzians are not sufficient to accurately model the lineshape at zero strain, and we need a minimum of three Lorentzians to reasonably fit the lineshape. One of these three peaks is related to $E^{\prime}$ mode while we associate the other two with the $L A$ branch and label them by $2 L A_{1}$ and $2 L A_{2}$. The description of the $L A$ branch as the sum of two Lorentzians has been made before in the case of MoS2 [38]. Such complex lineshapes are in general expected in double-resonance processes where one has to properly account for the phonon density of states as well as the electron-phonon couplings at different points in the Brillouin Zone. Our straindependent measurements help make the distinction between the $E^{\prime}$ and $2 L A$ bands as described below.

Shown in Fig. 3(a) are a sequence of Raman spectra of $\mathrm{WS}_{2}$ obtained at different values of strains. At each strain, we fit both the parallel and cross-polarization spectra to obtain a consistent set of peaks. Similar to WSe2, we find a splitting of the $E^{\prime}$ mode with applied strain. The measured Grüneisen parameter and shear deformation potential are $=0.54, \beta=0.14$ respectively. All extracted peaks positions, intensities and widths as a function of strain are shown in Fig. 3(b,c,d). Similar to WSe 2 , the $A^{\prime}$ mode shows a minimal response to strain due to 
its out of plane nature. Different from $\mathrm{WSe}_{2}$, the intensity of all the modes increases as a function of strain. Very interestingly, we find that at high strain $(>2.5 \%)$, the $A^{\prime}$ mode appears in cross polarization geometry while the P1 mode continues to be fully suppressed. We discuss these observations below.

To better understand the vibrational properties of monolayer $\mathrm{WS}_{2}$ and $\mathrm{WSe}_{2}$, we perform firstprinciples functional theory (DFT) calculations. In order to apply uniaxial strain, we use orthorhombic unit cell of different sizes, where a specific direction of the lattice, either armchair or zigzag is strained. Similar results were obtained at both directions, using a hexagonal cell (see figure S3 in Supplemental Material). Fig. 4(a and b) show the phonon dispersion curves for $\mathrm{WS}_{2}$ and $\mathrm{WSe}_{2}$ respectively at zero and finite strains. Vibrational modes are labelled accordingly to their symmetry at the $\Gamma$-point. It can be seen that there are six optical modes $\left(2 E^{\prime \prime}, 2 E^{\prime}, A_{1}^{\prime}, A_{2}^{\prime}\right)$ which are consistent with the $2 \mathrm{H}$ phase $\mathrm{D} 3 \mathrm{~h}$ space group symmetry. The observed phonon dispersion is consistent with previous theoretical studies [5, 10, 39-45]. With the application of uniaxial strain we observe a splitting of the doubly degenerate $E^{\prime}$ and $E^{\prime \prime}$ modes in both materials, due to reduction of the crystal symmetry. The variation in the frequency of the Raman active modes with applied strain is plotted in Fig. 4(c,d). All modes red shift with uniaxial strain with the exception of the $A^{\prime}$ mode in $\mathrm{WSe}_{2}$ which shows a variation of $0.03 \mathrm{~cm}-1$ per percent strain (see figures S4 and S5 in Supplemental Material). The variations in mode frequency for $\mathrm{WS}_{2}$ are consistent with the experimental results shown in Fig. 3(b). We also calculated the Grüneisen parameters from the phonon dispersion relations at different k-points using $\gamma=$ $-(V / \omega(q v))(\partial \omega(q v) / \partial V)$ (see figure S6 in the Supplemental Material). The results obtained for the $E^{\prime}$ modes are 0.45 and 0.54 for $\mathrm{WSe}_{2}$ and $\mathrm{WS}_{2}$, respectively, which are in remarkable agreement with the experimental results. Interestingly, these $\gamma$ values are well reproduced using 
a simple GGA approximation within the DFT functional. This is to be contrasted with other 2D materials such as $\mathrm{hBN}$, where the exchange-correlation potential has to be fine-tuned to achieve a quantitative agreement with experiment [46].

To study the second order Raman active modes, we examine phonon dispersions at the edges of Brillouin Zone. The frequencies of peaks $P_{1}$ and $P_{2}$ are found to be consistent with the transverse optical (TO) $E^{\prime \prime}$ and $E^{\prime}$ modes respectively [5]. To approximate the frequency of the 2LA mode we multiply the frequency of the $L A$ mode at the M-point of the Brillouin Zone by a factor of two, as this is a two-phonon process. An overall good agreement is seen between experimental and theoretical results with the exception of the $2 L A(M)$ mode of $\mathrm{WSe}_{2}$, which is increasing in wavenumber whereas experimental results show it to be decreasing. The smaller variation of this mode in the calculations is probably due to limitation in calculation of the second-order process.

In general, the intensity of a phonon mode in Raman spectrum depends both on the optical response of the material via the polarizability as well as the details of the electronic structure and electron-phonon coupling. All of these quantities evolve with applied strain. In our theoretical calculations, we examine the contribution of polarizability change with strain to the Raman intensity. In Fig. 4(e and f), the intensities of $A^{\prime}$ and $E^{\prime}$ modes show large increase in $\mathrm{WS}_{2}$, and small reduction in $\mathrm{WSe}_{2}$ instead. Both of these are consistent with the experimental observations. Our results for mode frequencies, anharmonicities and polarizabilities give us insight into the nature of the chemical bond between the metal and chalcogen atoms. $\mathrm{WS}_{2}$ has larger phonon frequency by about factor of two with higher anharmonicities, that would be simply expected for lighter chalcogen atom. However, we also see from experiment that its polarizability is several times larger than that of WSe2. This can be explained by comparing the ionic character of the W- 
$\mathrm{S}$ bond with that of the W-Se bond. A Bader analysis performed for both systems with various strains shows that the charge transfer towards the chalcogenide atoms in the W-S and W-Se bonds is -0.80 electrons/unit cell, and -0.18 electrons/unit cell, respectively, at $0 \%$ strain. These magnitudes increase by $3.5 \%$ and $16.07 \%$ as $3 \%$ strain applied into $\mathrm{WS}_{2}$ and $\mathrm{WSe}_{2}$, respectively. This difference in the ionic character of the two bonds and their response to strain provides a simple picture for the evolution of Raman spectra in these compounds with strain.

An intriguing finding in our Raman spectra is the presence of the $A^{\prime}$ mode in the crosspolarization geometry at high strain shown in Fig. 3(a). From the symmetry perspective, the $A^{\prime}$ mode is not cross-polarized Raman active at zero strain when the unit cell is hexagonal. However, upon the application of strain the symmetry is lowered from hexagonal to monoclinic (for a generic strain direction), making the $A^{\prime}$ mode observable in the Raman spectrum (see Supplemental Material). In general, we expect the intensity of this mode to increase with the size of the monoclinic distortion which is proportional to applied strain. We investigate this in our theoretical calculations applied to the cross polarization geometry with the monoclinic unit cell. At zero strain we indeed find a complete suppression of the $A^{\prime}$ mode. At a strain of $3 \%$, we observe a non-zero intensity for the $A^{\prime}$ mode, though its intensity is $99 \%$ lower in cross polarization when compared to the parallel polarization case. These results point to the role of large strains in actually modifying the symmetry of the lattice and thus changing selection rules. Such "strain engineering" is a promising avenue to tune the opto-electronic properties of both the semiconducting and metallic monolayer transition metal dichalcogenides. 


\section{FIGURES}
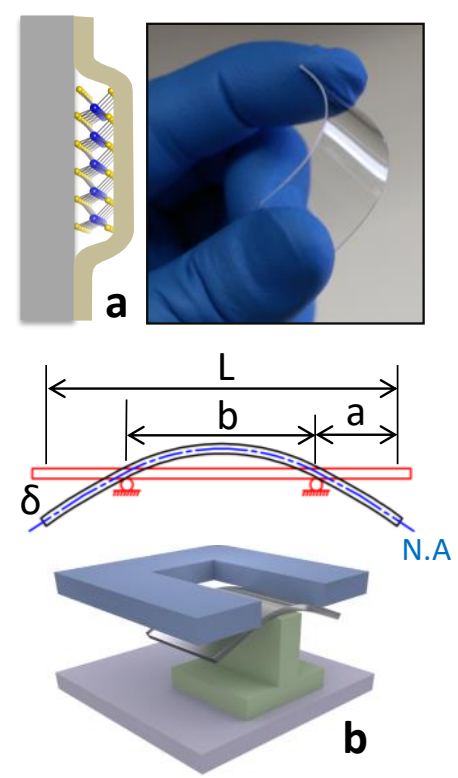
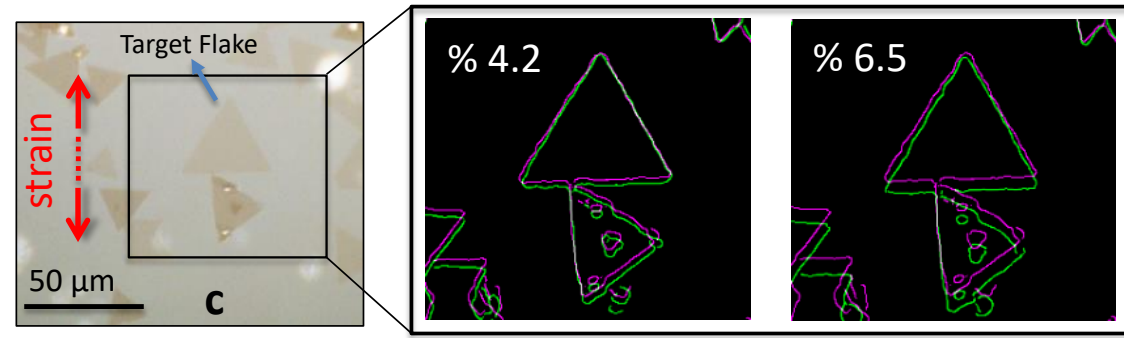

d

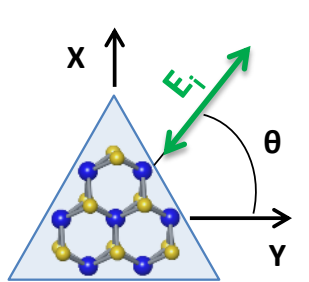

e

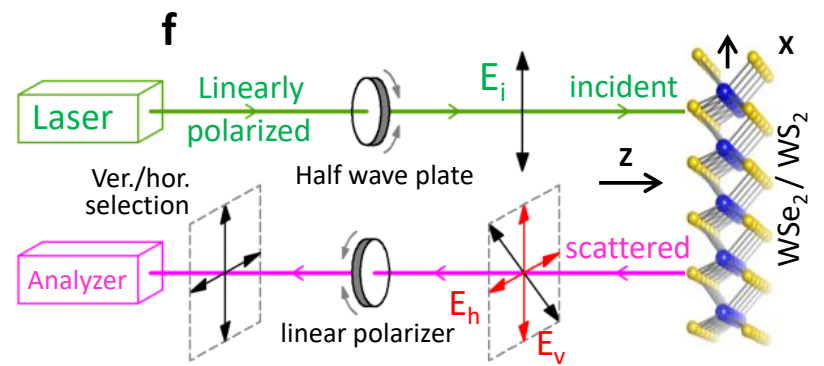

Figure 1. (a) Polymer encapsulated monolayer TMDs. (b) Strain apparatus. (c) Encapsulated WSe 2 monolayers. (d) Overlaid edgedetected images of strained(green) and unstrained(purple) monolayer edges for $4.2 \%$ and $6.5 \%$ calculated strains. (e) Incident light $(\mathrm{Y}, \theta=0)$ and strain $(\mathrm{X})$ directions with respect to crystal lattice. (f) Raman spectrometer setup. 


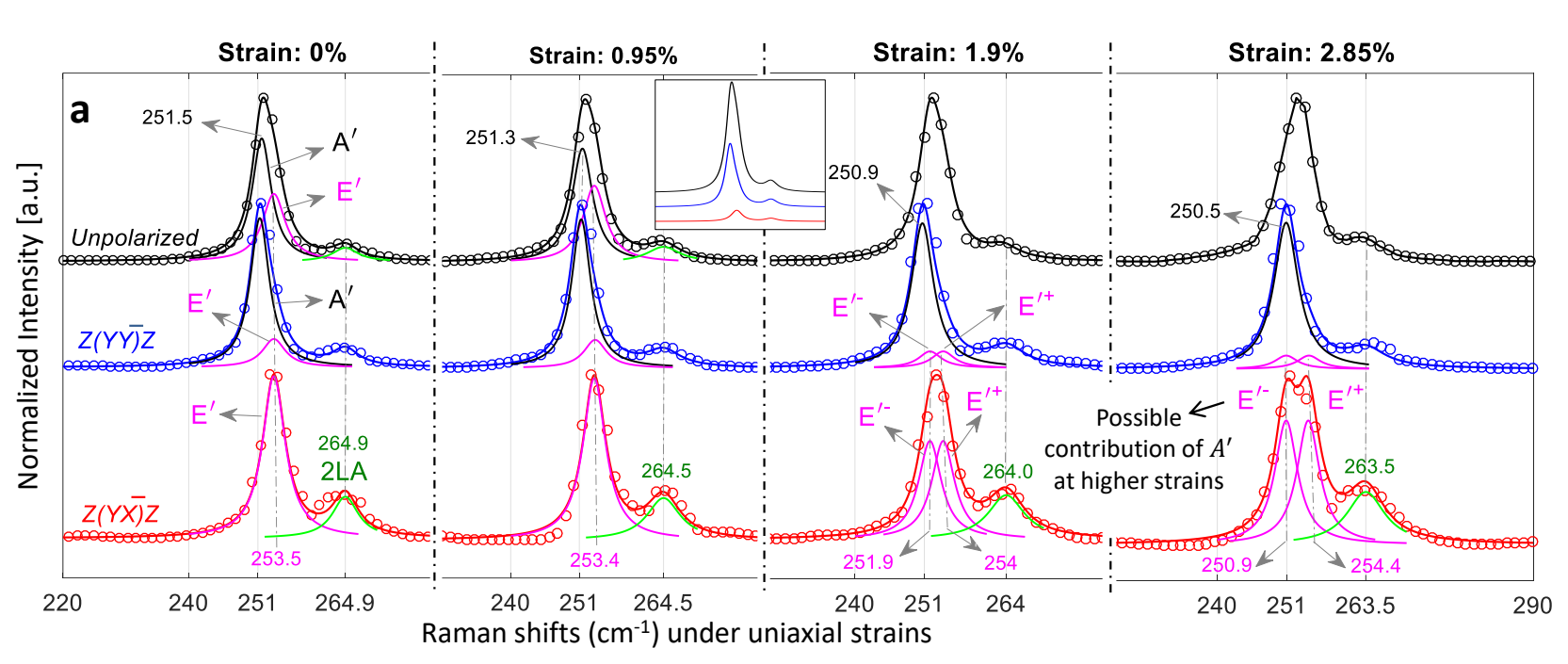

b

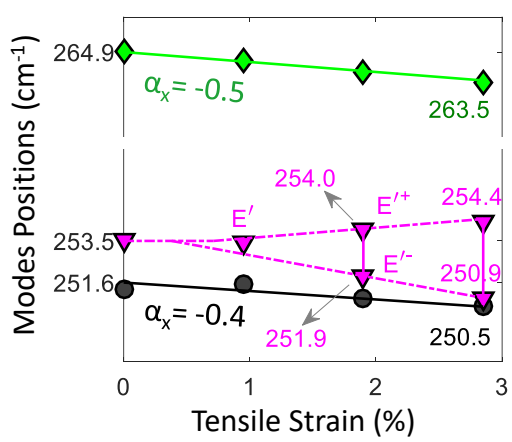

C

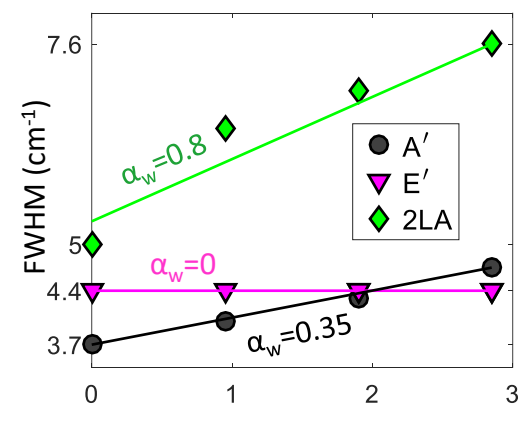

d

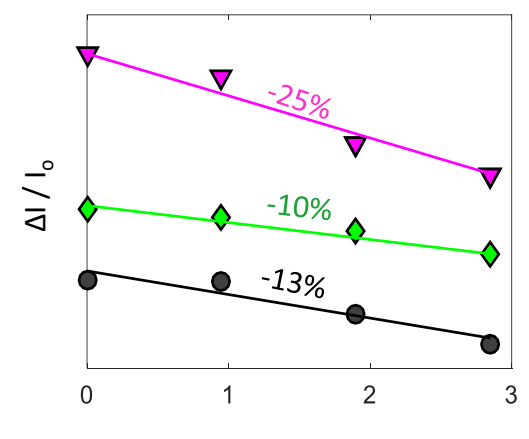

Figure 2. (a) Unpolarized (black), parallel-polarized (blue) and cross-polarized (red) Raman spectra of monolayer WSe 2 under various strains. The spectra are normalized to their peak intensities and shifted along y-axis for better illustration. Inset: real intensities of measured spectra without normalization. (b,c,d) Positions of phonon modes, Full Width at Half Maximum and relative intensity changes vs tensile strain. $\pm \alpha$ denotes the amount of change per percent tensile strain. 


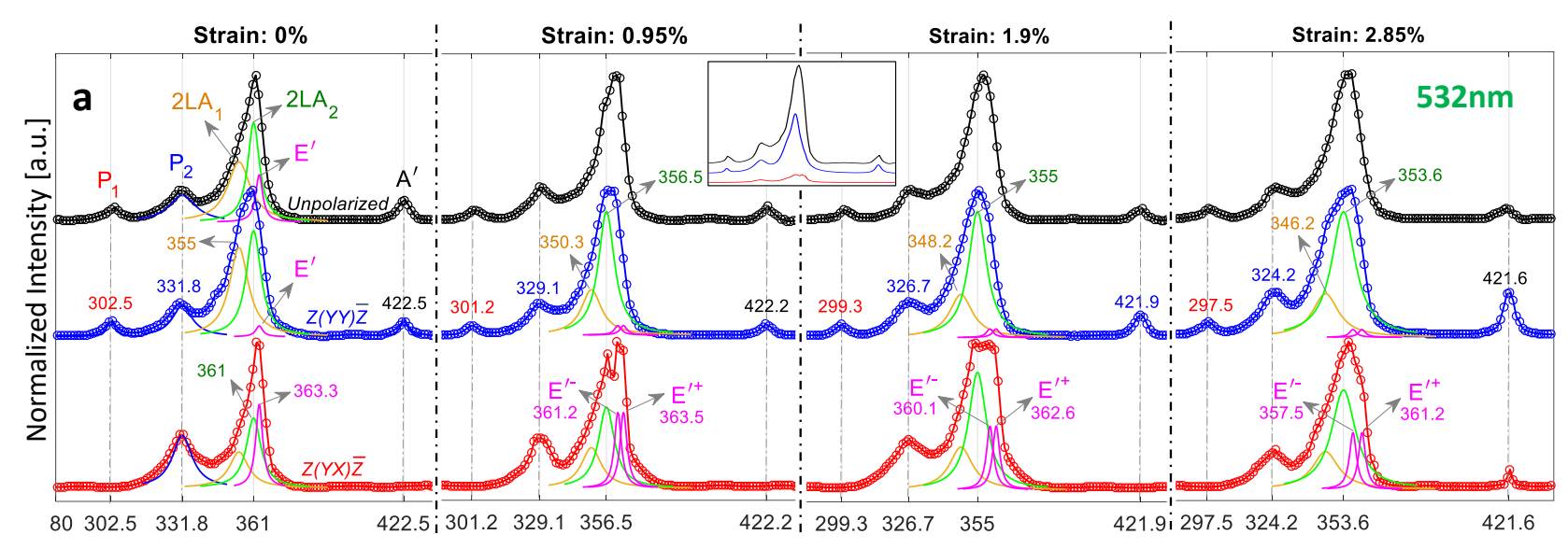

b
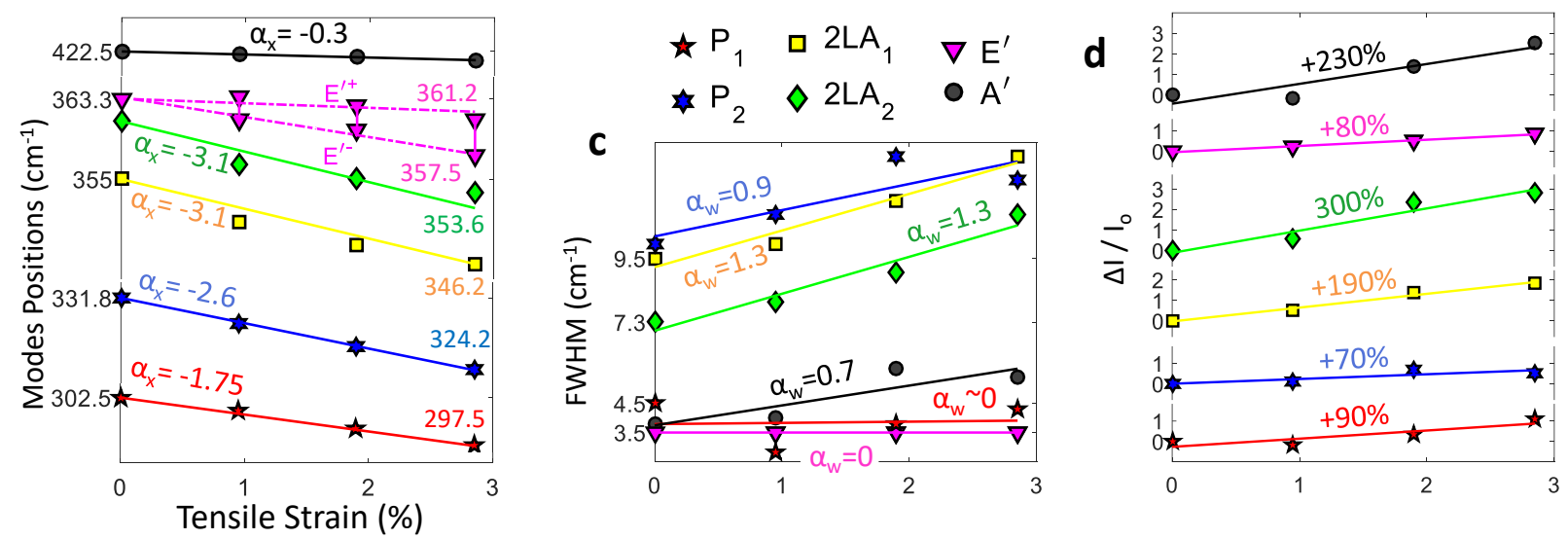

Figure 3 (a) Unpolarized (black), parallel-polarized (blue) and cross-polarized (red) Raman spectra of monolayer WS under $_{2}$ various strains. The spectra are normalized to their peak intensities and shifted along y-axis for better illustration. Inset: real intensities of measured spectra without normalization. (b,c,d) Positions of phonon modes, Full Width at Half Maximum and relative intensity changes vs tensile strain. $\pm \alpha$ denotes the amount of change per percent tensile strain. 

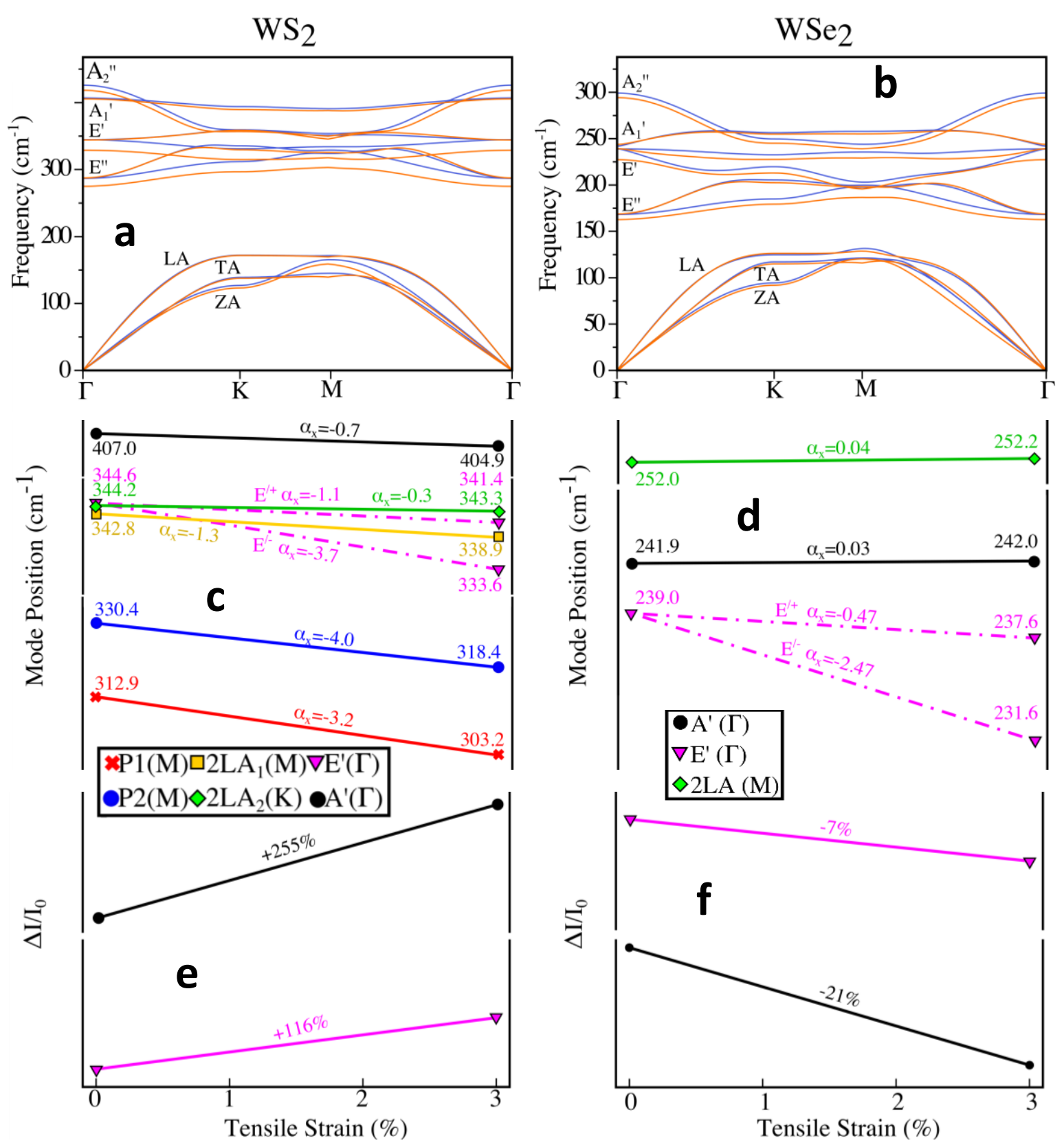

Figure 4 - Theoretical results on the vibrational properties of $\mathrm{WS}_{2}$ and $\mathrm{WSe}_{2}$ under strain. (a,b) Phonon dispersion curves. The blue and orange curves indicate unstrained $(0 \%)$ and strained $(3 \%)$ systems, respectively with the strain applied along armchair direction. Similar results have been obtained for zigzag directions (not shown). (c, d) Variation in mode frequencies with applied strain. In (c) P1 and P2 are defined at the M-point; and 2LA1 and 2LA2 are the M-point and K-point respectively, apart from E' and A' at $\Gamma$ point. In (d) $2 \mathrm{LA}$ is defined as M-point, and $\mathrm{A}^{\prime}$ and $\mathrm{E}^{\prime}$ at $\Gamma$-point. $\alpha$ shows curve slopes. Polarizations for $\mathrm{WS}_{2}$ and $\mathrm{WSe}_{2}$ are set on $\mathrm{A}^{\prime}$ at parallel; and E' at cross-polarized. (e, f) Relative intensity change of the Raman active modes at $\Gamma$-point 


\section{ASSOCIATED CONTENT}

\section{Supporting Information.}

The following file is available free of charge.

SupportingInformation.pdf (pdf file)

\section{AUTHOR INFORMATION}

\section{Corresponding Authors}

*Abhay N. Pasupathy: apn2108@ columbia.edu

* Elton Santos: e.santos@qub.ac.uk

\section{Author Contributions}

The manuscript was written through contributions of all authors. All authors have given approval to the final version of the manuscript.

\section{Funding Sources}

This work is supported by the Air Force Office of Scientific Research (grant number FA955016-1-0601, A.M.D.) and by the National Science Foundation (grant number DMR-1610110, A.N.P.). Shared characterization facilities were provided by the Materials Research Science and Engineering Center (provided through the NSF Grant: DMR-1420634). E.H.Y acknowledges NSF grant: ECCS-1104870, and Air Force Office for Scientific Research, grant: FA9550-12-10326. M.A.P thanks the Brazilian agencies Fapeming and CNPq for financial support. D.S 
thanks the studentship from the EPSRC-DTP award. E.J.G.S acknowledges the use of computational resources from the UK national high performance computing service, ARCHER, for which access was obtained via the UKCP consortium and funded by EPSRC grant ref EP/K013564/1; and the Extreme Science and Engineering Discovery Environment (XSEDE), supported by NSF grants number TG-DMR120049 and TG-DMR150017.

\section{ACKNOWLEDGMENT}

We thank Drew Edelberg, Nathan Finney and Nathan Zhao for experimental help, and James Hone and Jeffrey Kysar for discussions.

\section{REFERENCES}

1. Lee, C., et al., Elastic and frictional properties of graphene. physica status solidi (b), 2009. 246(11-12): p. 2562-2567.

2. Mohiuddin, T.M.G., et al., Uniaxial strain in graphene by Raman spectroscopy: G peak splitting, Gruneisen parameters, and sample orientation. Physical Review B, 2009. 79(20): p. 205433.

3. Huang, M., et al., Phonon softening and crystallographic orientation of strained graphene studied by Raman spectroscopy. Proceedings of the National Academy of Sciences, 2009. 106(18): p. 7304-7308.

4. Fang, W., et al., Strain-induced phonon shifts in tungsten disulfide nanoplatelets and nanotubes. 2D Materials, 2017. 4(1): p. 015007.

5. Zhang, X., et al., Phonon and Raman scattering of two-dimensional transition metal dichalcogenides from monolayer, multilayer to bulk material. Chemical Society Reviews, 2015. 44(9): p. 2757-2785.

6. Hosseini, M., et al., Strain-Induced Modulation of Electron Mobility in Single-Layer Transition Metal Dichalcogenides. IEEE Transactions on Electron Devices, 2015. 62(10): p. 3192-3198.

7. Peelaers, H. and C.G. Van de Walle, Effects of strain on band structure and effective masses in MoS2. Physical Review B, 2012. 86(24): p. 241401.

8. Cazalilla, M.A., H. Ochoa, and F. Guinea, Quantum Spin Hall Effect in Two-Dimensional Crystals of Transition-Metal Dichalcogenides. Physical Review Letters, 2014. 113(7): p. 077201.

9. Qian, X., et al., Quantum spin Hall effect in two-dimensional transition metal dichalcogenides. Science, 2014. 346(6215): p. 1344-1347.

10. Johari, P. and V.B. Shenoy, Tuning the Electronic Properties of Semiconducting Transition Metal Dichalcogenides by Applying Mechanical Strains. ACS Nano, 2012. 6(6): p. 5449-5456. 
11. Duerloo, K.-A.N., Y. Li, and E.J. Reed, Structural phase transitions in two-dimensional Mo- and W-dichalcogenide monolayers. Nat Commun, 2014. 5.

12. Duerloo, K.-A.N. and E.J. Reed, Structural Phase Transitions by Design in Monolayer Alloys. ACS Nano, 2016. 10(1): p. 289-297.

13. Song, S., et al., Room Temperature Semiconductor-Metal Transition of MoTe2 Thin Films Engineered by Strain. Nano Letters, 2016. 16(1): p. 188-193.

14. Duerloo, K.-A.N., M.T. Ong, and E.J. Reed, Intrinsic Piezoelectricity in TwoDimensional Materials. The Journal of Physical Chemistry Letters, 2012. 3(19): p. 28712876.

15. Wu, W., et al., Piezoelectricity of single-atomic-layer MoS2 for energy conversion and piezotronics. Nature, 2014. 514(7523): p. 470-474.

16. Roldán, R., et al., Strain engineering in semiconducting two-dimensional crystals. Journal of Physics: Condensed Matter, 2015. 27(31): p. 313201.

17. Conley, H.J., et al., Bandgap Engineering of Strained Monolayer and Bilayer MoS2. Nano Letters, 2013. 13(8): p. 3626-3630.

18. Desai, S.B., et al., Strain-Induced Indirect to Direct Bandgap Transition in Multilayer WSe2. Nano Letters, 2014. 14(8): p. 4592-4597.

19. Rice, C., et al., Raman-scattering measurements and first-principles calculations of strain-induced phonon shifts in monolayer MoS\$\{\}_\{2\}\$. Physical Review B, 2013. 87(8): p. 081307.

20. Wang, Y., et al., Strain-induced direct-indirect bandgap transition and phonon modulation in monolayer WS2. Nano Research, 2015. 8(8): p. 2562-2572.

21. Liu, Z., et al., Strain and structure heterogeneity in MoS2 atomic layers grown by chemical vapour deposition. Nature Communications, 2014. 5: p. 5246.

22. Yang, L., et al., Lattice strain effects on the optical properties of MoS2 nanosheets. Scientific Reports, 2014. 4: p. 5649.

23. Zhu, C.R., et al., Strain tuning of optical emission energy and polarization in monolayer and bilayer MoS2. Physical Review B, 2013. 88(12): p. 121301.

24. Amorim, B., et al., Novel effects of strains in graphene and other two dimensional materials. Physics Reports, 2016. 617: p. 1-54.

25. Castellanos-Gomez, A., et al., Local Strain Engineering in Atomically Thin MoS2. Nano Letters, 2013. 13(11): p. 5361-5366.

26. Dou, X., et al., Tuning and Identification of Interband Transitions in Monolayer and Bilayer Molybdenum Disulfide Using Hydrostatic Pressure. ACS Nano, 2014. 8(7): p. 7458-7464.

27. Ghorbani-Asl, M., et al., Strain-dependent modulation of conductivity in single-layer transition-metal dichalcogenides. Physical Review B, 2013. 87(23): p. 235434.

28. Guzman, D.M. and A. Strachan, Role of strain on electronic and mechanical response of semiconducting transition-metal dichalcogenide monolayers: An ab-initio study. Journal of Applied Physics, 2014. 115(24): p. 243701.

29. Kang, K.N., K. Godin, and E.-H. Yang, The growth scale and kinetics of WS2 monolayers under varying H2 concentration. Scientific Reports, 2015. 5: p. 13205.

30. Kang, K., et al., Graphene-Assited Anti-Oxidation of Tungsten Disulfide Monolayers: Susbtrate and Electric Field Effect. Advanced Materials, 2017. 
31. Godin, K., et al., Increased Monolayer Domain Size and Patterned Growth of Tungsten Disulfide through Controlling Surface Energy of Substrates". Journal of Physics D: Applied Physics, 2016. 49(325304).

32. Sahin, H., et al., Anomalous Raman spectra and thickness-dependent electronic properties of WSe $\$\{\}_{-}\{2\} \$$. Physical Review B, 2013. 87(16): p. 165409.

33. Tonndorf, P., et al., Photoluminescence emission and Raman response of monolayer MoS2, MoSe2, and WSe2. Optics Express, 2013. 21(4): p. 4908-4916.

34. Zhang, M., et al., Two-Dimensional Molybdenum Tungsten Diselenide Alloys: Photoluminescence, Raman Scattering, and Electrical Transport. ACS Nano, 2014. 8(7): p. 7130-7137.

35. O'Brien, M., et al., Mapping of Low-Frequency Raman Modes in CVD-Grown Transition Metal Dichalcogenides: Layer Number, Stacking Orientation and Resonant Effects. Scientific Reports, 2016. 6: p. 19476.

36. Robert, S., et al., Reversible uniaxial strain tuning in atomically thin WSe 2. 2D Materials, 2016. 3(2): p. 021011.

37. Cong, C., et al., Synthesis and optical properties of large-scale single-crystalline twodimensional semiconductor WS2 monolayer from chemical vapor deposition. arXiv:1312.1418, 2013.

38. Carvalho, B.R., et al., Intervalley scattering by acoustic phonons in two-dimensional MoS2 revealed by double-resonance Raman spectroscopy. Nature Communications, 2017. 8: p. 14670.

39. Berkdemir, A., et al., Identification of individual and few layers of WS2 using Raman Spectroscopy. Scientific Reports, 2013. 3: p. 1755.

40. Molina-Sánchez, A. and L. Wirtz, Phonons in single-layer and few-layer MoS\$\{\}_\{2\}\$ and WS\$\{\}_\{2\}\$. Physical Review B, 2011. 84(15): p. 155413.

41. Chang, C.-H., et al., Orbital analysis of electronic structure and phonon dispersion in MoS\$\{\}_\{2\}\$, MoSe\$\{\}_\{2\}\$, WS\$ \{\}$_{-}\{2\} \$$, and WSe $\$\{\}_{-}\{2\} \$$ monolayers under strain. Physical Review B, 2013. 88(19): p. 195420.

42. Terrones, H., et al., New First Order Raman-active Modes in Few Layered Transition Metal Dichalcogenides. Scientific Reports, 2014. 4: p. 4215.

43. Amin, B., T.P. Kaloni, and U. Schwingenschlog1, Strain engineering of WS2, WSe2, and WTe2. RSC Advances, 2014. 4(65): p. 34561-34565.

44. Zhan-Yu, W. and e. al, Effects of in-plane Stiffness and Charge Transfer on Thermal Expansion of Monolayer Transition Metal Dichalcogenide. Chinese Phys. B, 2015(24): p. 026501.

45. Peng, B., et al., Thermal conductivity of monolayer MoS2, MoSe2, and WS2: interplay of mass effect, interatomic bonding and anharmonicity. RSC Advances, 2016. 6(7): p. 57675773.

46. Cai, Q., et al., Raman signature and phonon dispersion of atomically thin boron nitride. Nanoscale, 2017. 9(9): p. 3059-3067. 
TOC GRAPHIC

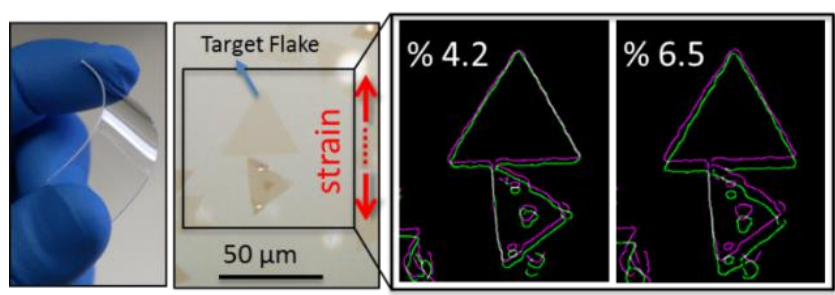

